\title{
Comparison of Indoor Localization Systems Based on Wireless Communications
}

\author{
Rejane Dalce, Thierry Val, Adrien Van Den Bossche
}

IRIT IRT, Université de Toulouse, Blagnac, France.

Email: dalce@irit.fr

Received June 21 ${ }^{\text {st }}, 2011$; revised August $19^{\text {th }}, 2011$; accepted September $2^{\text {nd }}, 2011$.

\begin{abstract}
Localization using a Wireless Sensor Network (WSN) has become a field of interest for researchers in the past years. This information is expected to aid in routing, systems maintenance and health monitoring. For example, many projects aiming to monitor the elderly at home include a personal area network $(P A N)$ which can provide current location of the patient to the medical staff. This article presents an overview of the current trends in this domain. We introduce the mathematical tools used to determine position then we introduce a selection of range-free and range-based proposals. Finally, we provide a comparison of these techniques and suggest possible areas of improvement.
\end{abstract}

Keywords: Wireless Sensor Networks (WSNs), Localization, Range-Based, Range-Free

\section{Introduction}

The modern world is centered on communication and speed. We want to be informed as quickly and reliably as possible about our health, the state of our environment, our current safety. Wireless Sensor Networks (WSNs) are a type of network that has been developed as a response to this need for control. Their nodes aim to become part of our environment and, using embedded sensors, help us monitor the status of relevant parameters.

As the needs evolved, the attention has turned to mobile sensors, introducing a new constraint: the collected data must be mapped to a specific location in order to ensure a correct interpretation of the values. Let's consider a mobile temperature sensor. When a measurement exceeds a set threshold, the system's reaction will depend on the current location of the node. Each predefined zone will be associated to an acceptable temperature range according to the usual activities taking place in this space. For example, during winter, detecting a temperature below $4^{\circ} \mathrm{C}$ in a bedroom hints a possible failure of the heater. On the other hand, the same value in a garage is perfectly normal. For the system to choose between alerting the system manager and ignoring the new value, the sensor's location must be available.

Position aware network nodes can also help in routing [1]. The benefits of localization being shown, the question is: How do we determine it?

We are not the first group to find this topic interesting.
Therefore, we plan on beginning with a description of the tools and methods generally used to solve the localization problem. We will focus on indoor solutions and ones that require minimum participation from the target. We will then introduce propositions that represent the current trends in location determination before identifying possible research areas and concluding this article.

\section{Classical Localization Algorithms}

When trying to localize an item, the first step is to define references. In this document, our references will be a subset of the network nodes. They will be aware of their location and will be referred to as "beacons". Another term frequently used for this purpose in the literature is "anchor".

The network's deployment area can be either indoor or outdoor. In the first case, the radio signal mainly suffers from attenuation and reflections. In the latter, setting up links on high distances and maintaining them becomes a great challenge.

Once the references have been established, relative location determination becomes possible. According to the implementation choices, this operation can be executed either by the mobile node or by a central node with greater processing power. When the mobile node is responsible for calculating its own position, an efficient algorithm must be used. The other solution requires the installation of at least one localization server who will process requests from all nodes in the network. 
The following subsections will introduce mathematical tools for positioning using 3 anchors as a reference.

\subsection{Trilateration}

This method requires the knowledge of the distance between the mobile and each beacon.

Let the anchors be $B_{1}, B_{2}$ and $B_{3} . M$ is the node we want to localize. The positions $\left(x_{i}, y_{i}\right)$ of the beacons as well as the distances $d_{\mathrm{i}}$ are known. The relationship between $M, B_{1}$ and $B_{2}$ can be written as:

$$
\begin{aligned}
& \left(x-x_{1}\right)^{2}+\left(y-y_{1}\right)^{2}=d_{1}^{2} \\
& \left(x-x_{2}\right)^{2}+\left(y-y_{2}\right)^{2}=d_{2}^{2}
\end{aligned}
$$

$M$ is therefore located at the intersection of circles $C_{1}\left(B_{1}, d_{1}\right)$ and $C_{2}\left(B_{2}, d_{2}\right)$. A third circle generated using the information of $\mathrm{B}_{3}$ enables us to choose the correct position between the two solutions of the general case of two circles intersecting (Figure 1).

\subsection{Triangulation}

In order to apply this method, the mobile must be aware of the distance between two beacons and the angles $\alpha$ and $\beta$ as shown in Figure 2. The law of sines yields:

$$
\begin{aligned}
& a=\frac{d^{*} \sin \alpha}{\sin (\pi-\alpha-\beta)} \\
& b=\frac{d^{*} \sin \alpha}{\sin (\pi-\alpha-\beta)}
\end{aligned}
$$

Once the characteristics of the triangle are known, the coordinates of the third summit can be calculated. As stated while describing trilateration, the third beacon or an additional hypothesis will allow identification of the correct solution.

\subsection{Fingerprinting}

This method is a pattern-recognition technique. It comprises to steps: in the first step or learning stage, some characteristics of the environment are measured at different locations and the data is stored along with a spatial

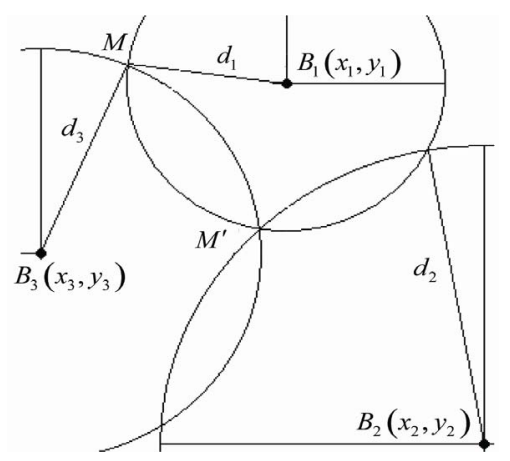

Figure 1. Trilateration. reference information. This step is also qualified as offline phase since it is usually performed before the activation of the localization service provided by the network.

The second step or online phase begins when the network is up and a mobile wants to determine its position. The same parameters are measured and the results are compared to the stored values.

The inherent modularity of fingerprinting enables researchers to work specifically on one phase or the other: hence the various propositions for selecting a position by comparing online and offline data.

According to the behavior of the measured characteristic through time, this method can be very interesting. Unfortunately, its cost in terms of setup time and data volume is high. Furthermore, any change in the configuration such as moving a beacon or modifying the environment, will imply creating a new database. In the case of a mobile WSN, the need for partial to complete updates will happen frequently.

\subsection{Multilateration}

Multilateration requires time information rather than distance. The time difference of arrival of a signal (TDOA) is used to determine position.

Figure 3 presents the case with the mobile being able to reach 4 beacons which are synchronized. The mobile node broadcasts a frame. Every beacon receiving the frame stores the local time of reception then calculates the difference between this time tag and the one provided by a set reference beacon. The collected time differences

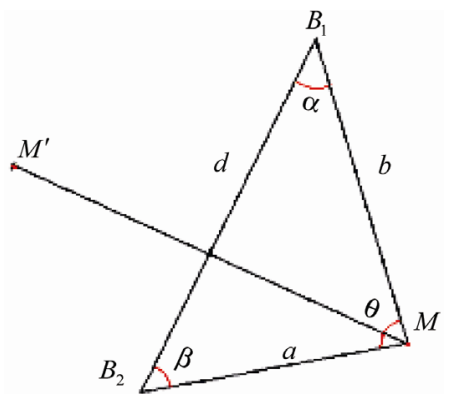

Figure 2. Triangulation.

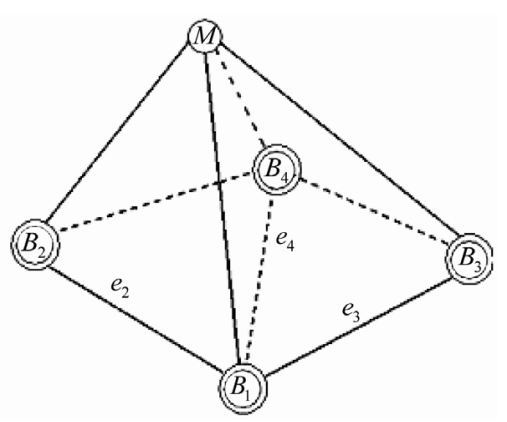

Figure 3. Multilateration. 
are used to write the following equations:

$$
\begin{aligned}
0= & c\left(t_{2}-t_{4}\right)-\frac{e_{2}^{2}}{c t_{2}}+\frac{e_{4}^{2}}{c t_{4}}+2 x\left(\frac{x_{2}}{c t_{2}}-\frac{x_{4}}{c t_{4}}\right) \\
& +2 y\left(\frac{y_{2}}{c t_{2}}-\frac{y_{4}}{c t_{4}}\right)+2 z\left(\frac{z_{2}}{c t_{2}}-\frac{z_{4}}{c t_{4}}\right) \\
0= & c\left(t_{2}-t_{3}\right)-\frac{e_{2}^{2}}{c t_{2}}+\frac{e_{3}^{2}}{c t_{3}}+2 x\left(\frac{x_{2}}{c t_{2}}-\frac{x_{3}}{c t_{3}}\right) \\
& +2 y\left(\frac{y_{2}}{c t_{2}}-\frac{y_{3}}{c t_{3}}\right)+2 z\left(\frac{z_{2}}{c t_{2}}-\frac{z_{3}}{c t_{3}}\right) \\
0= & c\left(t_{3}-t_{4}\right)-\frac{e_{3}^{2}}{c t_{3}}+\frac{e_{4}^{2}}{c t_{4}}+2 x\left(\frac{x_{3}}{c t_{3}}-\frac{x_{4}}{c t_{4}}\right) \\
& +2 y\left(\frac{y_{3}}{c t_{3}}-\frac{y_{4}}{c t_{4}}\right)+2 z\left(\frac{z_{3}}{c t_{3}}-\frac{z_{4}}{c t_{4}}\right)
\end{aligned}
$$

where :

$c$ : propagation speed

$\left(x_{i}, y_{i}\right)$ : beacon i coordinates

$e_{i}$ : distance from the chosen reference to node $B_{i}$. For the situation depicted in Figure $3, B_{1}$ is the reference beacon.

From a mathematical point of view, this equation system is easy to solve.

So far, we have browsed through methods that take as input a geometric relationship between the nodes and produce as an output the position of a node on a plane. Adding a beacon or a new measurement tool to the system would enable a $3 \mathrm{D}$ position to be identified.

We will now investigate the inputs which can be used in the context of radio communications.

\section{Radio Signal and Distance Measurement}

Having presented the main classical methods used to determine position using fixed reference nodes, we will now analyze the various radio signal characteristics that can be used for location determination purposes.

\subsection{Signal Power and Received Signal Strength Indication (RSSI)}

Due to interactions with the environment, a radio wave's energy decreases as it travels a certain distance. Many models describe the relationship between energy-loss and distance. Therefore they can be used to retrieve the spatial information.

\subsubsection{Free Space Model (Friis’ Equation)}

$$
\frac{P_{r}}{P_{t}}=G_{t} \times G_{r} \times \frac{1}{L} \times\left(\frac{\lambda}{4 \pi R}\right)^{2}
$$

Widely used in link budget estimation, the Free Space Model (Equation (8)) [2] expresses the impact of travelled distance $R$, gains $G_{t}$ and $G_{r}$ of the transmit and receive antennas, free-space losses L and signal frequency $f$ on the received power $P_{r}, P_{t}$ being the transmitted power.

\subsubsection{Two-Way Ground Reflection Model}

Whereas Friis' equation considers two antennas in Line of Sight (LOS) with only one signal path, Two Way ground reflection model gets closer to reality by taking into account a path due to signal reflection on the ground (Figure 4).

Deciding between the two models is done by examining the antenna heights and the distance $R$ between them. Let $D_{c}$ (Equation (10)), be the critical distance. When the spacing $\mathrm{R}$ is below $D_{c}$, the Free Space model is used. Otherwise, the two-way ground reflection model (Equation (9)) is employed.

$$
\begin{gathered}
\frac{P_{R}}{P_{T}}=\frac{G_{R} \times G_{T}}{L}\left(\frac{h_{T} \times h_{R}}{R^{2}}\right)^{2} \\
D_{c}=\frac{4 \pi h_{R} h_{T}}{\lambda}
\end{gathered}
$$

\subsubsection{Shadowing Model [3]}

This model comprises two parts:

1) Attenuation model: let $d_{0}$ be the reference distance and $P_{r}\left(d_{0}\right)$ the received power $d_{0}$. We use the following (Equation (11)) to calculate the received power at an arbitrary distance $d$ :

$$
\frac{P_{r}\left(d_{0}\right)}{P_{r}(d)}=\left(\frac{d}{d_{0}}\right)^{\beta}
$$

The attenuation factor $\beta$ is determined empirically.

2) Changes in power level at a fixed distance

Observation has revealed that a radio wave power's varies through time even when the measurements are taken at the same location. A log-normal probability law is typically used to represent these changes.

Considering these two aspects, the complete model is written as:

$$
\left[\frac{P_{r}\left(d_{0}\right)}{P_{r}(d)}\right]_{d B}=-10 \beta \log \left(\frac{d}{d_{0}}\right)+X_{d B}
$$

Although the Shadowing model is more complex, it is

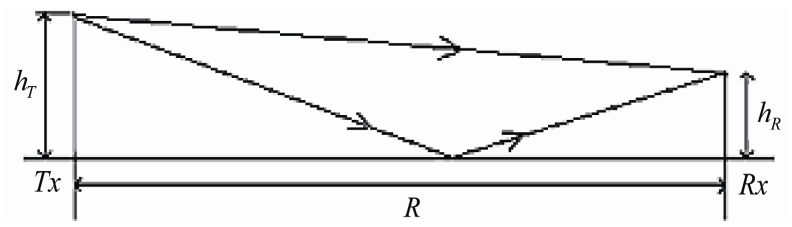

Figure 4. Propagation and reflection. 
the closest to reality as it does not consider the communication range as a circle or sphere but rather as a surface (volume) which shape changes through time, even without moving the source.

\subsubsection{RSSI}

Some physical layers give access to the raw power level information but almost all provide the RSSI. This parameter, usually a positive byte, informs about the received power level but a standard relationship between power and RSSI has not been defined. This function depends on the implementation. Therefore, a model based on data produced by platform $P$ might not be valid for platform $P^{\text {'. }}$

RSSI can also be used as an input to a fingerprinting method. In the case of beacons transmitting frames, the RSSI values are stored in a database along with the location where the measurement was taken and used during the online phase as a reference for position estimation.

RSSI-based localization is one of the most prolific themes since radio signal reception is the main requirement for its exploitation. Moreover, in the case where the mobile receives frames from beacons and processes them to extract its position, there is no Multi-User Interference problem to consider: two nodes receiving data do not disturb each other.

On the downside, the environment's effect is not null: the human body, due to the large proportion of water, attenuates the radio signal [4].

Using a model to match received power to distance requires configuration of the parameters. To perform this profiling, a measurement campaign is necessary. This step not only is an additional cost but is contrary to the concept and implementation of a spontaneous network.

\subsection{Time of Flight}

Two typical usages of Time of Flight (TOF) exist: use the TOF directly to calculate the distance or generate Time Difference of Arrival (TDOA) pairs.

Regardless of the location determination activities, message exchanges are to be expected in a network: taking advantage of this when designing the positioning system is a cost effective decision in terms of message generation. The time references needed will correspond to the reception and transmission time of messages. Let us illustrate this with a message exchange between $M$ and beacon $B$ (Figure 5). In the following description; $M$ and $B$ are synchronized.

Let $t_{a}$ be the $T x$ instant from $B$ and $t_{b}$ the $R x$ instant at $M$. Considering the fact that they share a common clock, the TOF is:

$$
t_{v o l}=t_{b}-t_{a}
$$

Two typical implementations are often encountered:

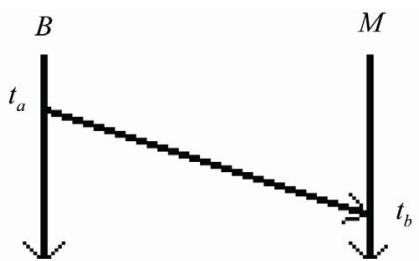

Figure 5. Time of Flight in a synchronized network.

\subsubsection{Single Signal}

In this case, distance is calculated as:

$$
\text { distance }=\text { vitesse }^{*} \text { temps }
$$

The speed being known, we only need to measure the duration. To obtain this value, we either consider a synchronized network or let each node measure local time information then combine them to obtain the TOF.

For example, in the situation depicted in Figure 5, the distance between $B$ and $M$ can be obtained by $c * t_{v o l}$.

\subsubsection{Combined Signals}

Sometimes, nodes in the network are equipped with another device which generates a signal which speed is different from that of the radio signal. It is then possible to obtain the distance from the difference in arrival time of the two signals. Assuming the two signals' emission started at the same time, the distance/time equation can be rewritten as (Equation (15)).

$$
\text { distance }=\tau *\left(\frac{v_{1}^{*} v_{2}}{v_{1}-v_{2}}\right)
$$

Parameters $v_{i}$ represent the signal speed of the two signals involved and $\tau$ corresponds to the measured TDOA.

Typically, a signal with lower speed is associated to the radio signal. Often, ultrasound is selected $\left(v_{\text {ultrasound }}=\right.$ $344 \mathrm{~m} / \mathrm{s}$ in air at $20^{\circ} \mathrm{C}$ ).

In any case, the nodes have to measure time. The results are influenced by the accuracy and precision of the measuring tools and also by the environment.

The first source of errors can easily be understood: if the smallest measurable time interval is a microsecond and the radio wave's speed is approximated at $3 \times 10^{8}$ $\mathrm{m} / \mathrm{s}$, the resolution of the distance measurement is $300 \mathrm{~m}$. This could be fine for measuring distance between very very large objects but is not acceptable for an indoor environment.

The indoor aspect is closely related to the second source of errors which is multipath. This term is used to designate the fact that, in an environment where signal reflection and diffraction exist, the receiver will detect multiple copies of the sent message with different delays and attenuation factors. In this context, identifying the first occurrence, hence the shortest path, can become a 
very complicated task.

Depending on whether the nodes are synchronized, the choice between the following TOF extraction procedures is straightforward:

1) In a shared clock configuration, the emission time of the signal is known to the receiver which can begin to generate at the same time its local version of the sequence. Correlating the two signals will allow estmation of the TOF as illustrated in Figure 6. Receiver and sender agreed on $t_{0}$ as the emission time of $S(t)$. Since the two nodes are separated by a certain distance, the transmitted version will reach the receiver with a delay $\tau$, which is proportional to the said distance. The value of $\tau$ maximize the correlation between the two signals.

2) In the case where each node has its own clock, a time interval will be associated with each node. This node in turn will be responsible for measuring the length of the interval and forwarding the data to the node requesting localization. We will explain this in much more detail as we present the location determination provisions of IEEE 802.15.4a [5].

\subsection{Angle of Arrival}

As introduced in 2.2, the angle of arrival (AOA) of the signal can also be used for location determination. Let's consider the mobile $\mathrm{M}$ as the source of the signal received by beacons $B_{1}\left(x_{1}, y_{1}\right)$ and $B_{2}\left(x_{2}, y_{2}\right)$. Angles $\alpha$ and $\beta$ are measured between the $\mathrm{MB}_{\mathrm{i}}$ axis and the perpendicular to the $B_{1} B_{2}$ direction.

In order to find M's position, let's define a coordinate system with $\mathrm{B}_{1}$ as the origin and $\left(B_{1}, B_{2}\right)$ as the y-axis.

The angles being known, finding the intersection of $\left(B_{1}, M\right)$ and $\left(B_{2}, M\right)$ is straightforward. $M$ 's coordinates (Figure 7) can be written as Equation (16).

$$
M\left(\frac{y_{2}}{\tan \alpha+\tan \beta} ; \frac{y_{2} \tan \alpha}{\tan \alpha+\tan \beta}\right)
$$

Solving the problem is therefore very simple. The only complicated matter is finding the angles. Usually sophisticated means like motorized antennas or antenna array are used. Care should also be taken during beacon place-

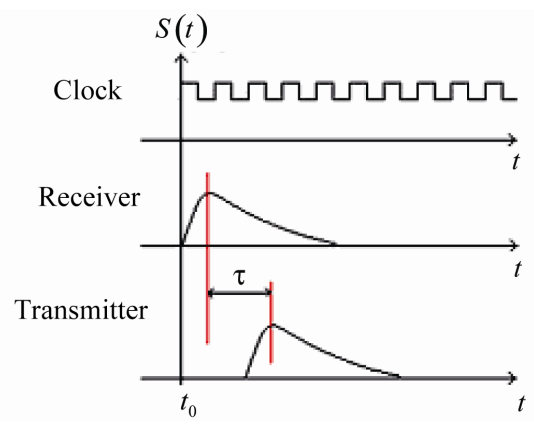

Figure 6. Time difference between local and received version.

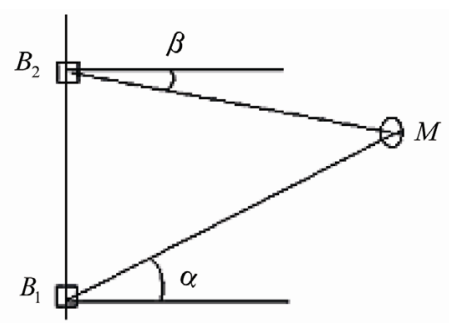

Figure 7. Angle of arrival and mobile position.

ment phase as reflected signals introduce errors in the angles, hence the position

\subsection{Signal Phase}

Signal phase is one of the most accurate means of measuring the distance. Let's consider our signal as a simple sine wave with wavelength $\lambda$. Let's also restrict our description to one cycle. When the measuring tool's output is $\theta$, it means the distance between receiver and transmitter is $\lambda^{*}$ :

$$
\lambda^{*}=\frac{\lambda \theta}{2 \pi}
$$

Therefore, if the signal frequency is $1 \mathrm{GHz}, \lambda$ will be $30 \mathrm{~cm}$ : $\theta$ ranging from 0 to $2 \pi, \lambda^{*}$, s limits will be zero and $30 \mathrm{~cm}$.

Measuring this angle means crosscorelating the received signal and a local version of the same signal. Therefore, at least during this part of the process, the nodes must be synchronized.

We must also keep in mind that this technique can only be used without an additional tool for distances below $\lambda$. Since we cannot differentiate $\theta$ and $\theta+2 k \pi, k \in \mathrm{N}$, we will either need a radio technology that implies a large $\lambda$ (as in low frequency techniques [6]) or add another method/set of tools that will allow us to solve the uncertainty.

\section{Localization in WSNs}

After exposing the mathematical tools and exploitable signal characteristics, we will present the additionnal constraints introduced by targeting a WSN and the various proposals that have been made.

\subsection{The Challenge}

A WSN is made of low-cost nodes with small processing power. A subset of nodes are aware of their location. The rest can be attached to a person or device and is used to collect information about this entity's motion and/or location.

In some situations, no maintenance plan is defined: Once deployed, new nodes can be added to the network but failing nodes are not repaired. Specifically, battery 
replacement is not an option: a node's lifetime is then equal to the battery's lifetime. Making the best out of this constrained resource implies energy-efficient components as well as energy-aware protocols and algorithms. However, we ought to mention studies in the field of energy-harvesting $([7,8])$ that could help lengthen the network's lifetime.

\subsection{The Constraints}

Calculating node position in a WSN means using the resources of the network for this purpose. As these resources are limited, the localisation algorithm will need to abide by the following constraints:

1) Energy: A node's lifetime can often be considered equal to that of its embedded battery. A protocol running on such module should be designed with this in mind: particularly, reducing the number of frames used and taking advantage of sleep modes are two common ways for the software to help conserve energy. Short-range technologies are another means of reducing power consumption as a longer range has a higher cost.

2) Environment: Most of the time, WSNs are deployed indoor. This implies a good resolution for the localisation system: the environment being made of rooms of size $n \times$ $p$ square meters $(10 \mathrm{~m}<n, p<100 \mathrm{~m})$, the system must be able to at least select one of the rooms and associate it with the mobile. The expected precision is also influenced as the results must render the decision possible.

3) Simplicity: Among the characteristics of WSN nodes is the low processing power. Therefore, the algorithms that will be implemented on these platforms must not require complex computations. This becomes a major concern when a fully distributed solution is envisioned.

4) Minimal intrusion: One field of application for localisation using WSNs is the monitoring of the elderly. In this case, the system must not depend on a specific action from the person carrying the module.

After this brief listing of the constraints imposed by the use of a WSN, we will present a few propositions that were made by the scientific community. This is not an extensive description but we believe it identifies the current trends in the location-determination field.

\subsection{Related Work}

Location determination techniques for WSNs can be classified in two groups: range-based techniques and range-free methods. This chapter will present the charac-teristics of each category and present a few propositions of each type.

\subsubsection{Range-Based Techniques}

As their name implies, range-based techniques use as input measurements taken by the system. In a WSN, it means signal characteristics being measured in real-time and fed to an entity that transforms it into a distance then a position.

These methods usually produce accurate results: if we recall the principles of signal phase-based distance measurement, in the appropriate situation, the accuracy is below the signal wavelength. The downside is the need to equip the nodes with specialized hardware, augmenting the cost and often size of the unit.

\subsubsection{UWB, 802.15.4a and TOF}

One of the basic characteristics of an UWB signal is having a bandwidth of at least $500 \mathrm{MHz}$ or a fractional bandwith (Equation (18)) greater than 20\%.

$$
B_{\text {fract }}=2 \times \frac{\left(f_{h}-f_{l}\right)}{\left(f_{h}+f_{l}\right)}
$$

with $f_{h}$ and $f_{1}$ being respectively the highest/lowest frequency in the band

The Spectral Power Density (SPD) being low, the disturbance caused to other systems using the same band is minimal. As a comparison, 802.11a has a DSP of 0.05 $\mathrm{W} / \mathrm{MHz}$ while UBW's is $6.67 \times 10^{-8} \mathrm{~W} / \mathrm{MHz}$. More information can be found in [9].

In Impulse-Radio UWB (IR-UWB), the pulse characteristic allow the existence of a wideband signal. The pulses (Figure 8) are typically Gaussian doublets (Equation (19)): being very narrow, they enhance signal resolution and render it immune to multipath (\$3.2). If the delay between the first signal and the copies is large enough, the first symbol can be correctly detected. In the context of localisation, it implies that measuring time by correlating the received signal to a locally generated sequence will produce a peak that can be clearly identified and used to indicate the TOF.

This spacing can be achieved either by spacing the pulses or by reducing the pulse width. The first solution has a negative impact on the radio link's bitrate. Therefore, the second has been chosen by IR-UWB.

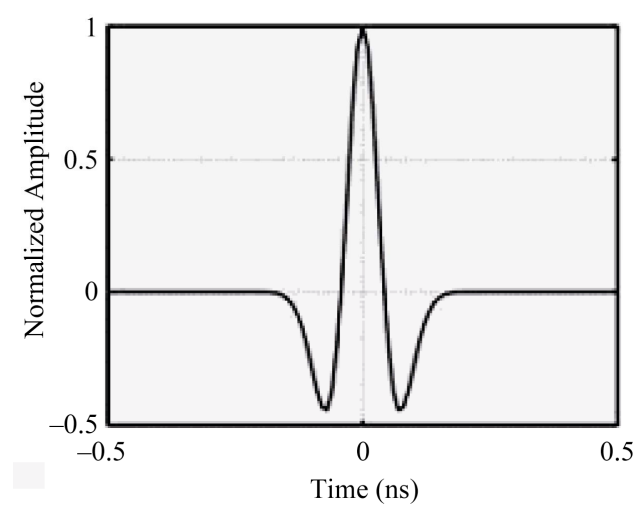

Figure 8. Gaussian doublet. 


$$
G(x)=\frac{1}{\sqrt{2 \pi \sigma^{2}}} \mathrm{e}^{\left(\frac{-x^{2}}{\sqrt{2 \sigma^{2}}}\right)}
$$

UWB technology is also promising in terms of low cost [10] and high bandwidth. The expected rates range from $110 \mathrm{Mbps}$ to $480 \mathrm{Mbps}$ and vary according to the emitter-receiver distance [9].

IEEE has recently updated its communication standard for low-power low-rate WSNs: one uses Chirp Spread Spectrum and the other one UWB. The latter's bands are $250-750 \mathrm{MHz}, 3.244-4.742 \mathrm{GHz}$ and $5.944-10.234$ $\mathrm{GHz}$. Just as in previous versions of 802.15.4, these bands are divided in channels [11]. Three channels are more than $1 \mathrm{GHz}$ wide and would be suitable for very accurate distance measurement.

A complete distance measurement using TOF is included in the standard. Network nodes that have the ability to measure TOF are designated as Ranging-capable DEVices (RDEVs). They must support a ranging counter, the ranging bit and the Ranging MARKER (RMARKER). These system functionalities will be described in detail as we explain the distance measurement process.

Common frames are used to measure time. The PHY header includes a special bit, the ranging bit, which purpose is to indicate that the current frame is a ranging frame (RFRAME) and is part of a measurement process. The actual time measurement uses the first pulse of the PHY header or RMARKER as a reference. No synchronization between the nodes is required. Figure 9 describes the message exchange.

The process begins with $\mathrm{RDEV}_{1}$ sending a frame to $\mathrm{RDEV}_{2}$ and storing the emission time, $T_{0}$, of the RMARKER of frame $_{1}$ (Figure 9). Upon reception, $\mathrm{RDEV}_{2}$ stores $T_{1}$ and replies to $\mathrm{RDEV}_{1}$, storing $T_{2}$. $\mathrm{RDEV}_{1}$ retrieves the fourth time information as it receives $\mathrm{RDEV}_{2}$ 's response. At this point, all relevant pieces of information have been collected but half is kept locally with each node since the frame is not generated

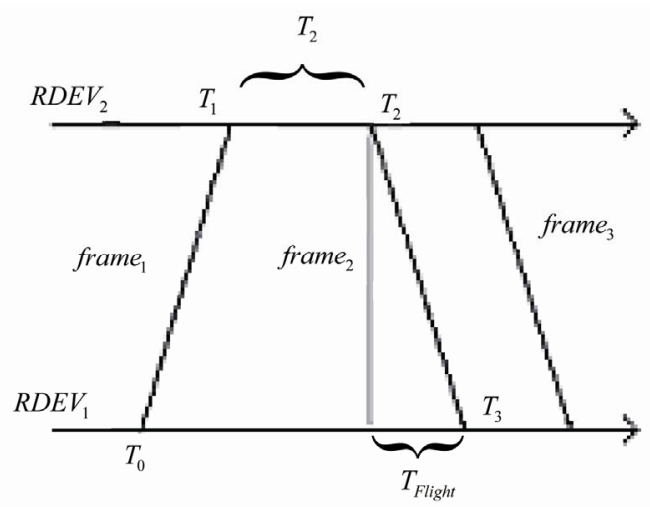

Figure 9. Two-way ranging (IEEE 802.15.4a). "on the fly". Since $\mathrm{RDEV}_{1}$ initiated the process, $\mathrm{RDEV}_{2}$ has to forward the data to $\mathrm{RDEV}_{1}$. The node will then be able to compute the TOF using (Equation (20)).

$$
T_{v o l}=\frac{T_{3}-T_{0}-\left(T_{2}-T_{1}\right)}{2}
$$

The ranging counter is the entity responsible for producing the $T_{i}$ values.

Although the clocks of both nodes are not synchronized, a certain level of accuracy is necessary. As the processing time of a frame ( $T_{a}$ in Figure 9) is in the order of the millisecond and the TOF for a $10 \mathrm{~m}$ distance is $30 \mathrm{~ns}$, errors in measuring $T_{a}$ will certainly and easily corrupt the results: at computation time, the nodes will combine time measurements that are not really similar (a second of time measured by node $i$ would be too different from a second of time measure by node $j$ ).

One means of solving this problem is finding the difference between the two clocks and using it to correct the results. The IEEE standard suggests extracting this information from the PHY layer; since a node needs to synchronize its clock to the sender's clock when decoding a frame, it means that the difference between the clocks is an available information.

This implies that the PHY layer must be able to forward this type of information to the upper layers. As this functionality might not be available, another technique is also proposed, which is Symmetric Double-Sided TwoWay Ranging (SDS-TWR) (Figure 10). In this case, the frame exchanges of Figure 9 are duplicated: the first part corresponds to Figure 9 and in the second part, the roles are reversed. This uses a special kind of frame, the acknowledgement for an acknowledgement: this enables the use of three frames for the two-measurements. Please note that the final data forwarding to $\mathrm{RDEV}_{1}$ is not illustrated.

Applying SDS-TWR reduces the difference between

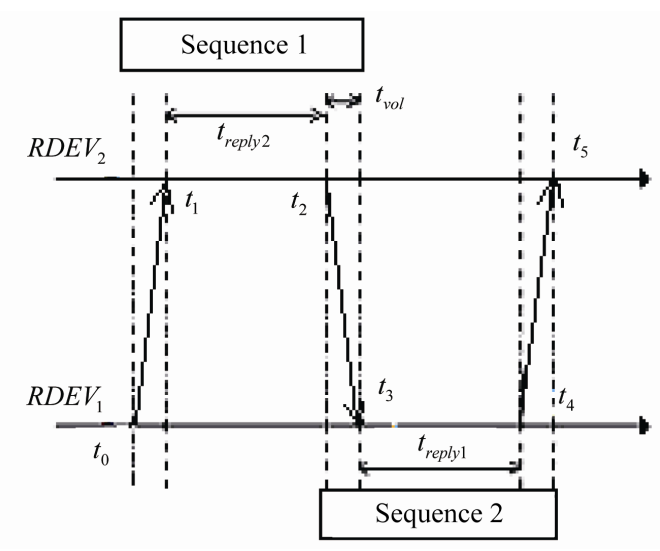

Figure 10. Symmetric double-sided two-way ranging (SDSTWR). 
the estimated value and the true TOF. Let $t_{\text {reply } 1}$ be the difference $t_{4}-t_{3}, t_{\text {reply } 2}$ be the difference $t_{2}-t_{1}, \Delta_{\text {reply }}$ the difference between $t_{\text {reply } 2}$ and $t_{\text {reply } 1}, t_{\text {vol }}$ the real value and $\hat{t}_{v o l}$ the estimated value. The accuracy goes from:

$$
\hat{t}_{v o l}-t_{v o l} \approx \frac{1}{2} \times t_{\text {reply } 2} \times\left(e_{1}-e_{2}\right)
$$

to:

$$
\hat{t}_{v o l}-t_{v o l} \approx \frac{1}{4} \times \Delta_{\text {reply }} \times\left(e_{1}-e_{2}\right)
$$

Accuracy can be further improved by taking into account the propagation delay induced by circuits between buffers and the antenna. The standard also suggest adding an auto-calibration feature to the node which would be responsible for evaluating this delay.

The IEEE 802.15.4a is a well detailed proposition but nevertheless, we must keep in mind that the localisation service is an optional functionality: not all 802.15.4acompatible devices will implement them.

\subsubsection{Fingerprinting}

One of the earliest propositions in the field of signal power fingerprinting is RADAR [12]. This system uses signal power and SNR to calculate position.

The first step, as described in section 2.3, is recording the power level of frames sent periodically by the mobile. These frames contain the current position and orientation of the mobile unit. During this offline procedure, the beacon nodes map each frame with the measured power and the time of reception. Since all the nodes have been synchronized, the time values are valid throughout the network.

As the offline phase ends, a database is built containing, for each mobile position and orientation (North, South, East, West), the measurements taken by each beacon. These measurements constitute the Signal Strength (SS) vector.

During the online phase, the beacon nodes keep a mean value of the measured signal level and use either empirical values contained in the database or a propagation model to determine position.

Nearest Neighbour in Signal Space (NNSS) method is used to search for the position using empirical data: this method selects as the solution the position for which the distance between the stored SS vector and the real-time SS vector is minimal. The results have been improved by only choosing the k NNSS and setting their centre of gravity as the position. This can be further enhanced by ignoring the orientation differences when choosing the $\mathrm{k}$ NNSS as this ensures physically different nodes.

Whereas RADAR used averaged RSSI values from the mobile, the work presented in [13] followed a different path and chose to consider the difference between RSSI values as their system's input. Since their solution is based on 802.11, we will use the term Access Point (AP) to refer to the beacon nodes.

The starting point of this work was the observation that the RSSI value returned was highly dependent on the measuring hardware (see 3.1.3). Therefore, the team first conducted an experiment with APs responsible for emitting frames and two different 802.11-compatible mobile nodes recording the RSSI values in a finite set of predefined positions. $A P$ pairs $\left(A p_{i}, A P_{j}\right)$ were then formed with the constraint of $i<j$. For each pair, the difference between RSSI values was computed. This hypothesis has been validated using real-world data: even though the values returned by the mobiles were not identical, the graphs showed the same global shape.

The impact of this hypothesis on localisation was determined by including it as a component of two wellknown methods: Nearest Neighbour (NN) and Bayesian Inference.

NN method uses a measurement database during the online phase as its reference. Each location in the database is associated to a point in the Signal Space: choosing a location as the estimated position is equivalent to finding the closest point in Signal Space to the measured RSSI. Through some adjustments, this method is made suitable to compute distance between RSSI differences rather than RSSI points.

Bayesian Inference is a means of choosing the most probable position given the current values. In order to become applicable to the problem at hand, an observation vector o must be defined and mapped to a position 1 . The contents of vector ok will be (AP pair ID, RSSI difference for the pair). Combining these values and the stored measurement yields:

$$
\operatorname{Pr}\left(o \mid l_{k}\right)=\prod_{1 \leq i<j \leq m} \operatorname{Pr}\left(d\left(A P_{i}, A P_{j}\right) \mid A P_{i} \times A P_{j}, l_{k}\right)
$$

The probability of being in the position referenced by $1 \mathrm{k}$ givent the current measured values is:

$$
\mathrm{P}_{\mathrm{r}}\left(l_{k} \mid o^{*}\right)=\frac{P_{r}\left(o^{*} \mid l_{k}\right) P_{r}\left(l_{k}\right)}{\sum_{k=1}^{n} P_{r}\left(o^{*} \mid l_{k}\right) P_{r}\left(l_{k}\right)}
$$

Maximizing this probability with respect to $l_{k}$ yields the true position.

The experiment conducted using off-the-shelf hardware has showed improved performance of the RSSIaugmented versions against the traditional implementations.

\subsubsection{TOF-Based Solutions}

The basic exploitation of TOF consist of measuring distances from the mobile to the beacons. As a result a vec- 
tor containing the various measured distances is obtained, along with the coordinates of each beacon. Position computation shows two main trends: using mathematical tools such as Maximum Likelihood (ML) methods to obtain the position and improving the performance of the PHY layer in order to achieve better accuracy.

ML methods' aim is to obtain the parameter value which maximizes the probability that the measurements are correct, given some system characteristics. The problem at hand can be written as:

$$
\hat{u}=\arg \max P_{u}(\hat{d} \mid u)
$$

$u$ : matrix containing beacon coordinates

$\hat{u}$ : estimated mobile position

$\hat{d}$ : measured mobile to beacon distances

Another method makes use of a cost function [14] such as Equation (26).

$$
F(x)=\sum_{i=1}^{N} \alpha_{i}^{2} f_{i}^{2}(x)
$$

with:

$$
f_{i}(x, y)=c \Delta_{t_{i}}-\sqrt{\left(x-x_{i}\right)^{2}+\left(y-y_{i}\right)^{2}}
$$

In these cases, a seed is required which should not be too far from the real solution in order for the algorithm to converge. Unfortunately, this type of computation requires a processing unit more powerful than a typical WSN node and therefore calls for a centralized solution.

In [15], the authors aim to calculate TOF between synchronized nodes without adding complex components or trying to counter clock phase differences. In order to mitigate multipath effect, the team proposed the use of the whole $2.4 \mathrm{GHz}$ band.

The method is comprised of two steps: unlike the fingerprinting methods, the first step is the online phase. The nodes use this phase to communicate then they turn off their transceivers and the offline phase begins. The sequence in which the actions are executed is regulated by the Event Clock (EC) which is present in each module. This clock's period, TEC, serves as an upper limit to the desynchronization between nodes.

The online phase can take place once synchronization has been attained: node A starts transmission of $\mathrm{k}$ copies of an n-symbol long ranging signal. Emission starts at the next rising edge of EC and the signal's duration is chosen equal to TEC. Node B turns on its receiver circuit two rising edges later and captures k-2 copies of the signal. After demodulation, B combines them in order to obtain one complete copy. Then B emits the received signal $\mathrm{k}$ times. When A has received the $\mathrm{k}-2$ versions, both modules turn off their transceiver.
The offline phase can then begin with each node performing a crosscorrelation between the received copy of the signal and a locally generated version. The ranging signal being a pseudonoise code (PN code) helps obtain a peak that indicates the time travelled between the nodes.

The prototype version of this system has been tested both indoor and outdoor and the accuracy was 1 - 3 meters depending on the environment.

\subsubsection{TDOA}

In [16], phase measurements and TDOA are combined in order to determine location in a synchronized network. The whole $2.4 \mathrm{GHz}$ ISM band is used to enhance accuracy: the signal's band increases from that of a single channel, $5 \mathrm{MHz}$, to $80 \mathrm{MHz}$ for the 16 channels defined: as the bandwidth increases, path separation and distance measurement are improved.

Four nodes are involved in the localisation process: two emitters, $T_{1}$ and $T_{2}$, and two receivers, $R_{1}$ and $R_{2}$. All nodes are aligned (Figure 11) and the receivers are included in both transmitters' range. Since the emitters are IEEE 802.15.4-based, their range will be $30 \mathrm{~m}$ and $\max \left(d\left(R_{1}, T_{2}\right), D\left(R_{2}, T_{1}\right)\right) \leq 30 \mathrm{~m}$, where $d(A, B)$ is the distance between two nodes $A$ and $B$.

The $T_{i}$ transmit simultaneously in two different channels chosen among the 16802.15 .4 channels.

The TDOA for each receiver is then computed. These values are referred to as $\Delta t_{1}$ and $\Delta t_{2}$ and written as:

$$
\begin{aligned}
& \Delta t_{1}=\tau_{21}-\tau_{11}+\tau_{t_{0}}=\tau_{0}+\tau_{t_{0}} \\
& \Delta t_{2}=\tau_{22}-\tau_{12}+\tau_{t_{0}}=-\tau_{0}+\tau_{t_{0}}
\end{aligned}
$$

The difference between emission time is termed $\tau_{\mathrm{t} 0}$ while $\tau_{0}$ is the time taken to travel the distance between $T_{1}$ and $T_{2}$. This distance, $d_{0}$, can be computed using a combination of these time information:

$$
d_{0}=c_{0} \tau_{0}=\frac{c_{0}}{2} \Delta \tau
$$

with:

$$
\Delta \tau=\Delta t_{1}-\Delta t_{2}
$$

From this we can see that as soon as the position of one of the transmitters is known, the other can easily be calculated.

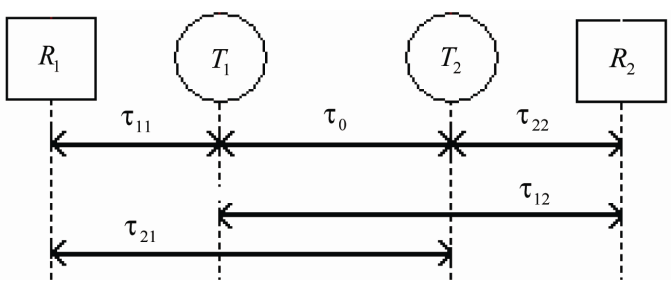

Figure 11. Node placement and time measurements. 
Phase measurements are also used in conjunction with the time measurements: as mentioned in 3.4, phase measurements are precise on one signal cycle. The longrange technology is then used to select a point and from then on, the phase measurements are used to refine the position/distance estimate.

\subsubsection{RSSI and Accelerometers}

The work presented in [17] combines RSSI-based fingerprinting to accelerometers values. The network is made of access points, beacons and mobiles. The APs receive broadcast frames from the beacons and the mobiles. A map is then generated, an RSSI vector being linked to a mobile at a certain position. This database is updated regularly, during the online phase. This compensates for the changes in the environment which might have an impact on the stored values.

When location determination is requested, the most probable position with respect to the APs' values is selected. Two search methods have been proposed: Maximum Search $\left(M_{x} S\right)$ and Minimum Search $\left(M_{n} S\right)$. The following points can help distinguish them from each other:

1) Search space: $M_{x} S$ determines the solution for each AP then computes the intersection. $\mathrm{M}_{\mathrm{n}} \mathrm{S}$, on the other hand, sets an initial solution as the possible points according to the AP which measured the maximum RSSI value. The subsequent tests use this set as their starting point, reducing the computations but augmenting the risk of a bad estimation, as the correct position might not be included in the initial solution.

2) Results: $M_{x} S$ yields better results than $M_{n} S$ but at a higher computational cost.

An interesting aspect of this proposition is the addition of accelerometers to the localisation system. They are used to record the number of paces executed by the user. Knowing the initial position, the user's motion characteristics and the accessible zones of the environment, the application can identify a possible zone of arrival.

When combined to $\mathrm{M}_{\mathrm{n}} \mathrm{S}$ and $\mathrm{M}_{\mathrm{x}} \mathrm{S}$, the accelerometerbased subsystem enhances the precision by $8 \%$. Nevertheless, the accelerometer information had to be ignored sometimes as their results did not match those of the RSSI.

\subsubsection{Range-Free Techniques}

The previous section introduced a handful range-based propositions. In the simplest case, access to the RSSI values was all it took to calculate the position. In a more complex example, a new physical layer had to be designed, synchronization between nodes was required before phase and time difference measurement could be taken.

Range-free methods aim to eliminate the need for ad- ditional hardware by relying on hypothesis about network connectivity. In this case, being cost effective takes a toll on system accuracy which will often be expressed as a percentage of the radio range.

\subsubsection{APIT}

APIT method [18] is based on the Point In Triangulation test. It enables a node to determine whether or not it is located inside a given triangle. If any displacement of the node gets it closer to/farther from at least one of the triangle summits, then the node is inside the triangle (Figure 12(a)). If it gets farther from/closer to all summits at the same time, then the node is outside the triangle (Figure 12(b)).

This description works in the mathematical world but in real-life, some approximations must be done. The node's movement is not always a parameter that can be controlled by the system: as we stated earlier, the solution should avoid depending on a specific user behaviour. Hence, the node uses the relationships between it's neighbours and the beacons that act as triangle summits to investigate the situation. The test can then be written as: if all the node's neighbours are closer to at least one of the beacons, then the node is inside the triangle (Figure 12(c)). This version of the test is likely to fail when the node placement is irregular or part of the neighbours are outside de triangle (Figure 12(d)).

The network is comprised of beacons or anchor nodes which are aware of their position and have a powerful transceiver, and mobile nodes which use APIT to determine their position. APIT is an area-based algorithm because its aim is to obtain a surface where the node is most likely to be.

The mobile nodes collect the frames transmitted by the

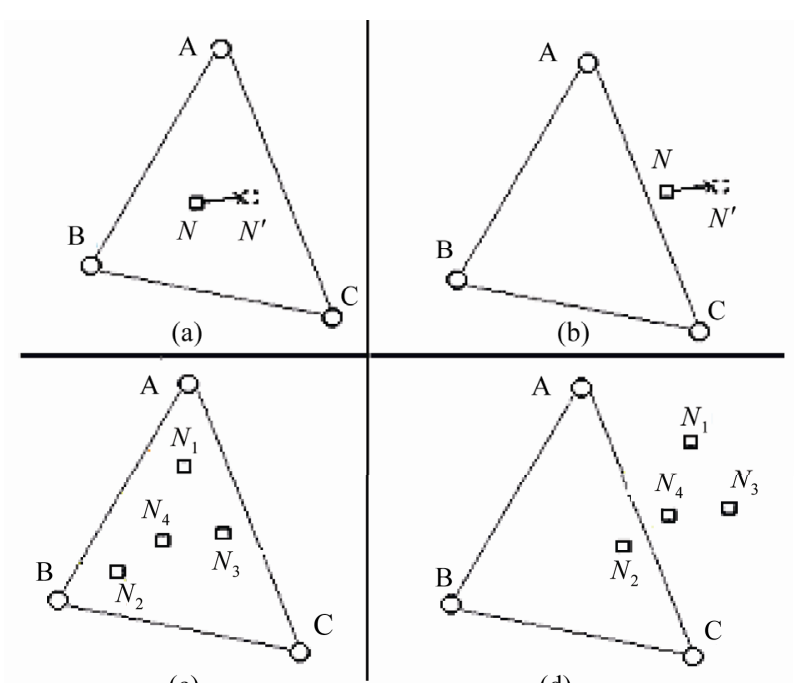

(c)

(d)

Figure 12. Mathematical and network forms of APIT algorithm. 
anchors in order to build a list of in-range beacons. The node then selects groups of three beacons and runs the test. This process is repeated until no more groups can be formed or a certain surface-size threshold is reached, whichever happens first.

The node also uses a matrix that represents the environment: each cell corresponds to a zone and a zone is part of one of the triangles. Every successful run of the test leads to the incrementation of the value contained in all the cells corresponding to the surface of the selected triangle. Upon failure, the value is decreased by one.

In the end, the surface with the highest associated values is selected as the area and its centre of gravity is assumed to be the node's true position..

\subsubsection{DV-Hop Suite}

DV-Hop is a popular range-free algorithm. Its original version along with a few proposed enhancements are presented in this subsection.

\subsection{Original DV-Hop}

DV-Hop [19] is a method often cited as a reference in range-free propositions. It considers mobile nodes as unknowns and uses a logic similar to that of distance vector routing protocols to find the position. Each beacon broadcasts a frame containing its true position and a field dedicated to hop counting. Each node that receives this frame stores the hop count value, increments the field and forward the frame if the hop count is lower that the previously known value for the specified link. Once the beacons have collected these data, they compute a mean hop size. For example, in Figure 13, beacon $A_{1}$ can mathematically calculate its true distance to $A_{2}$ and $A_{3}$. The hop count for each link is also obtained via flooding. Estimating hop size makes use of the following equation:

$$
\text { hopsize }_{1}=\frac{d_{1,2}+d_{1,3}}{\text { hop }_{1,2}+\text { hop }_{1,3}}
$$

with $d_{i, j}$ being true distance between beacons $i$ and $j$, $h o p_{i, j}$ representing the number of hops between beacons $i$ and $j$

This hop size is flooded to the network. The mobile nodes will use it to estimate the distance between itself and the anchor (Equation (33)).

$$
d_{i, x}=\text { hopsize }_{i} \times \text { hopcount }_{i, x}
$$

where hopcount $t_{i, x}$ is the number of hops between mobile $x$ and beacon $i$. Once the distances to three beacons are known, trilateration can be applied.

By using the hop count mechanism, DV-Hop aims to enable location determination on mobile nodes that are out of range of beacons.

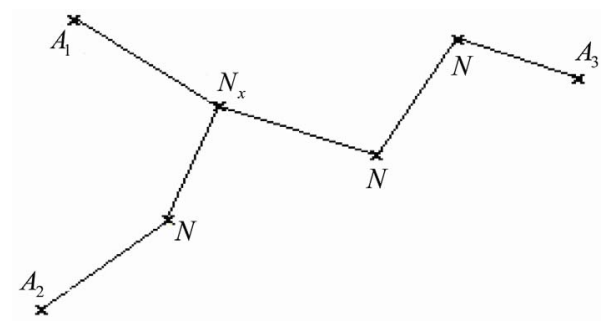

Figure 13. DV-Hop.

\subsection{Constraint DV-Hop}

Many groups have tried to improve DV-Hop. We will first mention Constrained DV-Hop or CDV-Hop, which is described in [20].

The proposal stemmed from the fact that an implicit relationship exists between the communication range $\mathrm{D}$ and the distance calculated by DV-Hop. When a node's hop count to a beacon is 1 , it implies that the distance is lower than $1 * D$. If the spacing is 2 hops, the distance is then bounded by $2 * D$. The new system will therefore take these constraints into account and it can be rewritten as:

$$
\min \sum_{i=1}^{M}\left(\left(x_{i}-x_{u}\right)^{2}+\left(y_{i}-y_{u}\right)^{2}-d_{i}^{2}\right)^{2}
$$

with

$$
\sqrt{\left(x_{v_{j}}-x_{u}\right)^{2}+\left(y_{v_{j}}-y_{u}\right)^{2}} \leq D, L=1
$$

where

$$
\begin{gathered}
D \leq \sqrt{\left(x_{v_{j}}-x_{u}\right)^{2}+\left(y_{v_{j}}-y_{u}\right)^{2}} \leq D L, \\
L \neq 1, j=1,2, \cdots, M_{1}
\end{gathered}
$$

This proposal was validated using simulations of a hundred nodes, 82 out of which were mobiles. The deployment area was $50 \times 50$ square meters. Various tests were conducted with different mobile to beacons rations and values of $D$. In the end, they all indicated that CDV-Hop was a definite improvement to DV-Hop.

\subsection{Checkout DV-Hop}

Also aiming to improve DV-Hop is the work described in [21]. Mobile nodes in this case are divided in two classes: level 1 mobiles can communicate with 3 beacons or more while level 2 nodes do not. Depending on its class, a node will choose which algorithm to use in order to obtain its position.

Level 1 nodes will refer to the Mid-Perpendicular Method: In the case of three anchors, we will consider the mid-perpendicular of each side of the beacon triangle. The node will assume their intersection to be its position. this solution only requires knowledge of the coordinates of two beacons. 
The improvement is more noticeable when we consider the case of level 2 nodes. The Checkout DV-Hop, as its name implies, integrates a new step to the classical algorithm. The closest anchor, $A_{\text {near }}$, in terms of hopcount, corresponds to the link for which the smallest error accumulation will occur during path length estimation. The node can then use the distance between itself and $A_{\text {near }}$ to improve the position obtained with DV-Hop.

Figure 14 shows the effect of Checkout DV-Hop. True node position is $N_{x}$ but according to DV-Hop, the node is located in $N_{D V \text {-Hop }}$. Checkout DV-Hop modifies the estimated position by substracting the $A_{\text {near }}$ to $N_{x}$ distance from the $A_{\text {near }}-N_{D V-H o p}$ axis, leading to a new estimated position, $N_{\text {checkout }}$.

The new method, 2RaLA, has been compared to other methods such as Centroid (\$4.3.2.3) + DV-Hop and Convex Position Estimation (CPE) [22] + DV-Hop using MATLAB. Under different configurations, 2RaLA has produces better results than the other algorithm combinations.

\subsubsection{Centroid and Weighted Centroid}

Centroid [23] assumes spherical isotropic propagation of the radio signal as well as identical range for all nodes in the network. The beacon nodes form a regular mesh and broadcasts frames every $\mathrm{T}$ seconds without collisions.

Each mobile receiving these frames computes a connectivity metric using (Equation (37)).

$$
C M_{i}=\frac{N_{\text {recv }}(i, t)}{N_{\text {sent }}(i, t)} \times 100
$$

where:

$N_{\text {recv }}(i, t)$ : number of frames emitted by beacon $i$ and received during interval $t$

$N_{\text {sent }}(i, t)$ : total number of frames emitted by beacon $i$ during interval $t$

According to a predefined threshold, the mobile node selects a subset of beacons to take into account when calculating its position. The coordinates of this group of beacons will then be used to compute their centre of gravity, which the mobile node will assume to be its position. Figure 15 is an example of the results of the process for a non regular-mesh of beacons. The estimated position is represented by a plus sign while the dots correspond to the beacon nodes.

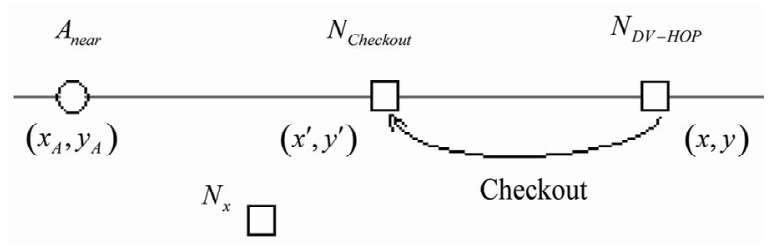

Figure 14. Checkout DV-Hop.

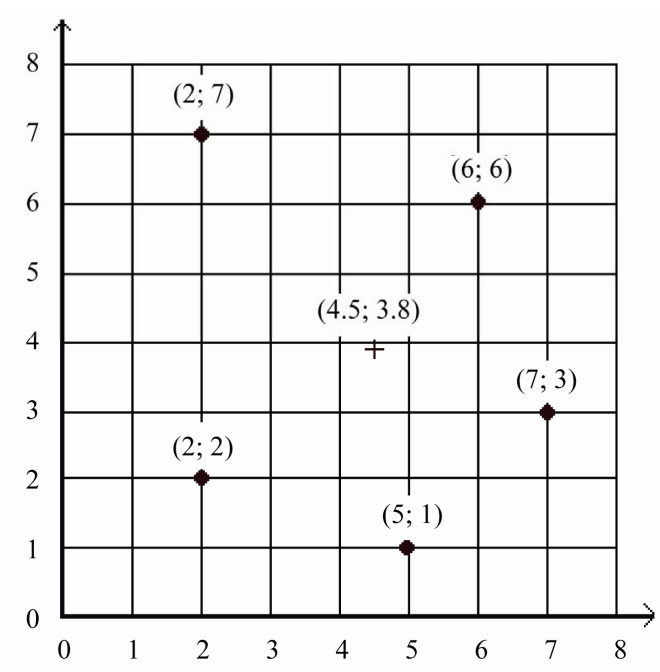

Figure 15. Localizing with Centroid.

Centroid has been validated in an outdoor setup: four beacons have been used to localize in a $10 \times 10$ square meters, leading to a mean error of $2 \mathrm{~m}$.

Improvements such as Weighted Centroid Localization (WCL) [24] exist. WCL adds the RSSI information to the process by associating weights to the links between the mobile node and the beacons. This estimated position is then written as:

$$
P=\left(\frac{\sum_{j=1}^{n} w_{i j}(d) x}{\sum_{j=1}^{n} w_{i j}(d)}, \frac{\sum_{j=1}^{n} w_{i j}(d) y}{\sum_{j=1}^{n} w_{i j}(d)}\right)
$$

where $P=P(x, y)$ is the estimated position, $\left(x_{B i}, y_{B i}\right)$ are the coordinates of beacon $i$ and $w_{i j}(d)$ (Equation (39)) is the weight associated with the link.

$$
w_{i j}(d)=\frac{1}{R S S I_{\text {stand }}^{q}}
$$

The weights are calculated as follows; the mean RSSI value for each beacon is determined then, as the prototype returned a negative value in the range $[-110 \mathrm{~dB},-50$ $\mathrm{dB}]$, translated to positive values using (Equation (40)).

$$
R S S I_{\text {strand }}=-(R S S I+49)
$$

This RSSIstand then takes its values in the interval [1 $\mathrm{dB}, 51 \mathrm{~dB}]$.

As expected, the results have shown that, with the addition of weights, the estimated position moved closer to beacons with higher weight.

Although RSSI is exploited in this proposition, we did not consider it as range-based since its usage here corresponds neither to a power to distance model, nor to a power to position mapping. 


\subsubsection{Hybrid Solutions}

Range-free and range-based methods do not always oppose each other. In fact, they sometimes combine their strenghts to bring novel solutions combining real-time measurements to network-wide hypothesis.

\subsubsection{HexNet}

In Hexnet [25], the environment was subdivided in hexagons (Figure 16). Two types of nodes are available and three behaviours can be implemented. The network begins with a fixed node called anchor which has two emitters: one is very directional while the other is omnidirectional. Every anchor is aware of its position and together, the anchors of the network form a regular grid. This type of node is not energy constrained and its communication range $R$ is greater than that of the sensor nodes.

The second category comprises the sensor nodes. They can act as backbone sensors or as simple sensors depending on their relative position to the anchor.

The anchor's position defines the centre of the first hexagon. The subsequent hexagons are defined in a pattern that ensures all polygons are adjacent.

For each new $h_{k}$, a backbone sensor (BS) is selected. The sensors that do not implement the BS behavior assume their own position to be that of the centre of gravity of all heard BSs.

The anchor is responsible for selecting the sensor that will act as the BS for the hexagons adjacent ot its own. From then on, the peripheral BSs will manage the BS election process.

First, nodes must be aware of their angular position in the coordinate system defined by the anchor and an arbitrary direction that will serve as the $\mathrm{x}$-axis. The anchor

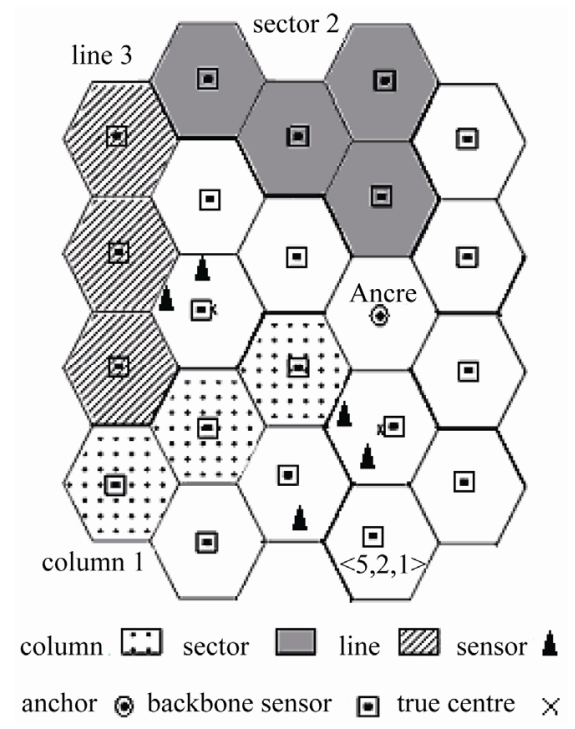

Figure 16. HexNet network. uses its directional antenna and, while rotating it slowly, broadcasts the current angle. When $\mathrm{BS}_{\mathrm{k}}$ has to choose the $\mathrm{BS}_{\mathrm{i}}$ for one of its adjacent hexagons, it can reduce the number of sensors involved by first announcing the angle corresponding to the hexagon. Along with the angle of interest, the $\mathrm{BS}_{\mathrm{k}}$ sends data about the geometrical relationship between itself, the target and the anchor.

Once the candidates have received these informations, they estimate the distance between themselves and the centre of the hexagon. This result serves as an initial value for a local timer: the node with the smallest result will consider itself as the new BS upon expiration of the timer.

Hexnet therefore enables localization using a single anchor, given the specific hardware requirements are met.

\subsubsection{GPS and Mobility Pattern}

The idea in [26] is to combine GPS-enabled nodes to a mobility pattern in order to localize nodes in an outdoor environment. The beacons would be equipped with GPS receivers and would be synchronized. Their position can change through time.

These beacons are responsible for broadcasting regularly the time of their last GPS update, their position and the time between reception of GPS information and current emission.

These data are stored by the mobiles along with the local reception timestamp in the Anchor History Table (AHT). The GPS coordinates (Longitude, latitude) are converted to seconds and their size is reduced to ease computations. The mobile then uses this to create its own mobility pattern, rewriting its longitude and latitude as functions of time. Once the mobility pattern has been defined, the mobile will estimate its position periodically, regardless of reception of frames from the beacons.

In this localisation system, GPS is used to synchronize the beacons and also to enable for position correction after the mobile has left the network for some time. While out of coverage, the mobile will keep estimating its position using a model which is probably not valid. As soon as the mobile re-enters the network, the received frames will help rectify the estimations.

This proposition has been evaluated through both simulation and prototype. The simulation involved 400 nodes placed on a $500 \times 500$ square meters and was used to examine the effects of node speed, beacon ration and AHT size.

In the prototype configuration, the mobile was connected to a laptop which acted as a data sink while the node moved on a $100 \times 100$ square meters surface.The experiment revealed the impact of weather, through GPS, on the prototype. It also indicated that sudden change in 
movement would induce erroneous predictions. The results also showed a localization around of roughly $10 \mathrm{~m}$ with 5 beacons seen by the mobile per minute.

\section{Summary and Comparison}

The previous sections were devoted to general methods and specific propositions from the research community. The current chapter will focus on comparisons, first of the means of measuring offered by WSNs then on the various propositions presented in section 4.

\subsection{Signal Characteristics Comparison}

We will begin with the comparison of the signal characteristics usable for localisation in a WSN.

Table 1 lists six means of obtaining positioning information using radio communications. The best with respect to accuracy are TOF-UWB and signal phase. Their performance make them really interesting for location determination purpose but the hardware is not always simple to obtain. In the case of UWB, very few companies offer chips that feature distance measurement functionality. For example, Decawave [27] intends to launch its $802.15 .4 \mathrm{a}$ compatible chip but mass production is planned for 2012. Until then, the per chip price keeps testing out of the reach of many.

Phase measurement imply adding expensive modules to the system. Because of the angular uncertainty, it must be coupled with another long range technique. Furthermore, the node placement must abide by the LOS constraint or the measured phase will corrupt the computation results. This dependence upon node placement is also a factor to consider when designing an AOA-based localisation system.

Although the time information retrieved using narrow band technologies cannot compare to the data UWB is expected to produce, it still ranks higher than RSSI. Distance estimation using signal power indication suffers from environment obstructions and its own variations through time. In order to attain precise measurements with RSSI, a fingerprint database with an automatic and cost-effective update system must be created. Regardless of its insufficiencies, RSSI will still spawn new propositions as the information is available on almost every hardware implementation, unlike TOF.

Just as easy to obtain is hopcount. Originally, this was a metric used by Distance-Vector routing protocols but its ease of use has enabled its usage in the field of localisation. Unfotunately, in the case of nodes which do not form a regular grid, the results of distance estimation through hopcount are most unreliable.

\subsection{Propositions Comparison}

Before diving in the comparison of the various rangebased and range-free propositions, let us explain the criteria that have been chosen. We will evaluate the localisation algorithms based on environment constraints, whether they are decentralized, the computational requirements and the accuracy.

One of the first aspects to consider is whether the system operates indoor or outdoor: it indicates how well the proposed system manages in case of obstructions.

Some solutions assume there will be a special node with high processing power which will be responsible for all calculations. This node will be termed the localisation server. This type of centralized architecture allows for very low-cost mobile nodes but the failure of the server implies the unavailability of the localisation service. Also, the strain on the nodes closest to the server cannot be neglected as there is a need for data forwarding to and from the server. A decentralized solution will ensure computation is not dependant on a single node but will require all the nodes to be upgraded.

The fourth criterion is accuracy but sometimes, we will take into account the improvements brought by the proposition. These benefits will have to be considered in conjunction with the computational constraints, i.e. the conditions that must be satisfied for the proposed method to be applied.

Table 1. Comparison of ranging means.

\begin{tabular}{|c|c|c|c|}
\hline Signal Criterion & Accuracy & Cost & $\begin{array}{l}\text { Environment } \\
\text { (constraints, degradations...) }\end{array}$ \\
\hline $\begin{array}{l}\text { TOF } \\
\text { UWB }\end{array}$ & centimeter & $\begin{array}{l}\text { Synchronization } \\
\text { Hardware }\end{array}$ & High frequency attenuation \\
\hline $\begin{array}{l}\text { TOF } \\
\text { Narrow band technology }\end{array}$ & Meter & $\begin{array}{l}\text { Hardware } \\
\text { Synchronization }\end{array}$ & Multipath \\
\hline Signal phase & $<$ wavelenth on one cycle & Hardware & Line Of Sight \\
\hline RSSI/Power & Meter and varies through time & $\mathrm{X}$ & Human \\
\hline $\mathrm{AOA}$ & -- & Hardware & Multipath \\
\hline Hopcount & Radio range & $\mathrm{X}$ & $\mathrm{X}$ \\
\hline
\end{tabular}


Since the goal is location determination, a critical parameter will be its performance. In the cases where a prototype has been built, real-world data is available and make the comparison easier. For example, the results for [15] are far better than those of RADAR. [15] being based on TOF, the impact of the measuring tool is not to be overlooked. On the other hand, since both of them are centralized methods, [15] is more cost-effective than RADAR since the amount of data necessary to execute localisation is minimal: [15] calculates a crosscorrelation whereas RADAR operates with an extensive RSSI-locantion database. Finally, [15] has been tested both in indoor and outdoor environments.

In [16], a method for calculating position in a 1D problem has been provided using TDOA and phase measurement in a synchronized network. Since the un- derlying measuring tools are among the bests according to Table 2, the performance of this technique is quite impressive. Nevertheless, we find it rather complicated for a 1D problem, especially when all nodes can hear each other. Generalization to the 3D situation is expected to involve a dense network or invoque an astute solution.

The comparison becomes more difficult when we turn to range-free methods, especially when evaluating the accuracy. Due to the assumption of "hardware independency", these propositions are most of the time only simulated. Centroid stands out as real-world performance information is provided. The attained precision is similar to that of RADAR while requiring 4 anchors.

An interesting characteristic is the fact that most of these solutions are distributed: each node is responsible for collecting the data related to its situation and estimate its position. Using simple operations makes attaining this goal easier.

However, the assumptions on the network configuration are not trivial: even if it is not always clearly mentioned, having an isotropic network is not to be overlooked. It implies either accepting non negligible errors or ensuring a regular grid of beacons.

If we consider the whole table, we can identify two tendencies: in the range-based context, both data collection and processing are costly. On the other hand, rangefree methods take advantage of simple computations while assuming a very constrained node deployment.

\section{Conclusions}

The ideal WSN is expected to allow fast node deployment and propose a light infrastructure. Combining properties from range-based and range-free techniques would enable the production of solutions closer to this goal: Range-based's ability to obtain a localization information without placing tight constraints on the deployment pattern of the beacons would make the network more spontaneous while a distributed approach would benefit from the simplicity of the processings proposed in range-based techniques. At the same time, range-based methods could enhance the reliability of the estimates generated by range-free techniques.

Using complementary hardware/software components could also improve the performance of the location determination system by providing supplementary information related for example to the mobile's motion. Having an internal datasource would also reduce the strain on the

Table 2. Propositions' summary.

\begin{tabular}{|c|c|c|c|c|c|}
\hline & & $\begin{array}{l}\text { Indoor VS } \\
\text { outdoor }\end{array}$ & $\begin{array}{l}\text { Centralized VS } \\
\text { Décentralized }\end{array}$ & $\begin{array}{l}\text { Computational } \\
\text { constraints }\end{array}$ & Accuracy/improvements \\
\hline \multirow{3}{*}{ 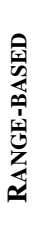 } & RADAR & Indoor & Centralized & Synchronized beacons & $3-4.3 \mathrm{~m}(50 \%)$ \\
\hline & TOA [15] & Tested in both environments & Centralized & $\begin{array}{l}\text { Crosscorrelation } \\
\text { Sensor synchronization }\end{array}$ & $1 m_{R M S}-3 m_{R M S}$ \\
\hline & $\begin{array}{l}\text { TDOA phase } \\
\text { [16] } \\
\text { 1D solution }\end{array}$ & Indoor & Distributed & $\begin{array}{l}\text { Sensors synchronization } \\
\text { Sensors placed on a line } \\
\text { New PHY layer }\end{array}$ & $\begin{array}{l}16 \mathrm{~cm} \text { (phase) } \\
79 \mathrm{~cm} \text { (TDOA) }\end{array}$ \\
\hline \multirow{3}{*}{ 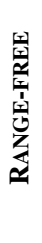 } & DV-HOP & & Distributed & Isotropic node placement & \\
\hline & CENTROID & Outdoor & Distributed & Isotropic beacon placement & $\begin{array}{l}2 \mathrm{~m} \text { mean error with } 4 \text { bea- } \\
\text { cons }\end{array}$ \\
\hline & APIT & Unspecified & Distributed & $\begin{array}{l}\text { Isotropic node placement } \\
\text { High beacon number } \\
\text { Border effect }\end{array}$ & Similar to DV-Hop \\
\hline 罢 & HEXNET & unspecified & Centralized & $\begin{array}{l}\text { Rotating antenna (anchor) } \\
\text { Isotropic placement of anchors }\end{array}$ & Single anchor \\
\hline
\end{tabular}


radio communication subsystem as the period of the precise location determination operation could then be increased.

Finally, range-based solutions implemented in a completely distributed fashion and propositions exploiting combined signals from various sources remain a field in which research still has improvements to bring.

\section{REFERENCES}

[1] R. Want, A. Hopper, V. Falcão and J. Gibbons, "The Active Badge Location System," ACM Transactions on Information Systems (TOIS), Vol. 10, No. 1, 1992, pp. 91102. doi: $10.1145 / 128756.128759$

[2] W. Fahs, "Diffusion d'Informations Partagees Entre Mobiles Cooperants Evoluant Sous une Meme Cellule d'un Reseau Sans fil Avec Infrastructure," Ph.D. Dissertation, Université de Clermont-Ferrand, 2009.

[3] T. S. Rappaport, "Wireless Communications, Principles and Practice," 2nd Edition, Prentice Hall, Upper Saddle River, 1996.

[4] D. Lieckfeldt, J. You and D. Timmermann, "Characterizing the Influence of Human Presence on Bistatic Passive Rfid-System," Wireless and Mobile Computing, Networking and Communications, Marrakech, November 2009, pp. 338-343.

[5] Specific Requirements Part 15.4: Wireless Medium Access Control (MAC) and Physical Layer (PHY) Specifications for Low-Rate Wireless Personal Area Networks (WPANs) Amendment 1: Add Alternate PHYs, IEEE Standard for Information Technology Telecommunications and Information Exchange between Systems, August 2007.

[6] F. Evennou, "Techniques et Technologies de Localisation Avancées Pour Terminaux Mobiles dans Les Environnements Indoor," Ph.D. Dissertation, Université Joseph Fourier, Grenoble, 2007.

[7] J. Rabaey, F. Burghardt, D. Steingart, M. Seeman and P. Wright, "Energy Harvesting-A systems Perspective," International Electron Devices Meeting, Washington, December 2007, pp. 363-366.

[8] http://en.wikipedia.org/wiki/Energy_harvesting

[9] M. Ghavami, L. B. Michael and R. Kohno, "Ultra Wideband Signals and Systems in Communication Engineering," 2nd Edition, John Wiley \& Sons Ltd., New York, 2007. doi:10.1002/9780470060490

[10] T. K. K. Tsang and M. N. El-Gamal, "Ultra-Wideband (UWB) Communications System: An Overview," 3rd International IEEE-NEWCAS Conference, August 2005, pp. 381-386.

[11] L. De Nardis and M.-G. Di Benedetto, "Overview of the IEEE 802.15.4/4a Standards for Low Data Rate Wireless Personal Data Networks," Workshop on Positioning, Navigation and Communication, Hannover, April 2007, pp. 285-289.

[12] P. Bahl and V. N. Padmanabhan, "RADAR: An In-Build- ing RF-Based User Location and Tracking System," Nineteenth Annual Joint Conference of the IEEE Computer and Communications Societies, Vol. 2, 2000, pp. 775784.

[13] F. Dong, Y. Chen, K. Li and D. Guo, "A Calibration-Free Localization Solution for Handling Signal Strength Variance," Mobile Entity Localization and Tracking (MELT), Vol. 5801, 2009, pp. 79-90.

[14] H. Liu, H. Darabi, P. Banerjee and J. Liu, "Survey of Wireless Indoor Positioning Techniques and Systems," IEEE Transactions on Systems, Man and Cybernetics, Part C: Applications, and Reviews, Vol. 37, No. 6, 2007, pp. 1067-1080.

[15] S. Lanzisera, D. T. Lin and K. S. Pister, "RF Time of Flight Ranging for Wireless Sensor Network Localization," Workshop on Intelligent Solutions in Embedded Systems (WISES), Vienna, June 2006, pp. 1-12. doi:10.1109/WISES.2006.329127

[16] S. Schwarzer,M. Vossiek, M. Pichler and A. Stelzer, "Precise Distance Measurement with IEEE 802.15.4 (ZigBee) Devices," IEEE Radio and Wireless Symposium, Orlando, March 2008, pp. 779-782.

[17] Z. Liang, I. Barakos and S. Poslad, "Indoor Location and Orientation Determination for Wireless Personal Area Networks," International Workshop on Mobile Entity localization MELT, Vol. 5801, 2009, pp. 91-105.

[18] T. He, C. Huang, B. M. Blum, J. A. Stankovic and T. Abdelhazer, "Range-Free Localization Schemes for Large Scale Sensor Networks," Proceedings of the 9th Annual International Conference on Mobile Computing And Networking, September 2003, pp. 81-95.

[19] D. Niculescu and B. Nath, "Ad Hoc Positioning System (APS)," Global Telecommunications Conference, Vol. 5, November 2001, pp. 2926-2931.

[20] W.-W. Ji and Z. Liu, “An Improvement of DV-HOP Algorithm in Wireless Sensor Networks," International Conference on Wireless Communications, Networking and Mobile Computing, Wuhan, September 2006, pp. 1-4.

[21] L. Gui, A. Wei and T. Val, "A Two-Level Range-Free Localization Algorithm for Wireless Sensor Networks," 2010 6th International Conference on Wireless Communications Networking and Mobile Computing (WiCOM), Chengdu, September 2010, pp. 1-4.

[22] L. Doherty, K. S. J. Pister and L. El Ghaoui, "Convex Position Estimation in Wireless Sensor Networks," Twentieth Annual Joint Conference of the IEEE Computer and Communications Societies, Vol. 3, 2001, pp. 1655-1663.

[23] N. Bulusu, J. Heidemann and D. Estrin, "GPS-Less LowCost Outdoor Localization for Very Small Devices," IEEE Personal Communications, Vol. 7, No. 5, 2000, pp. 28-34. doi:10.1109/98.878533

[24] F. Reichbach and D. Timmermann, "Indoor Localization with Low Complexity in Wireless Sensor Networks," IEEE International Conference on Industrial Informatics, Singapore, August 2006, pp. 1018-1023. doi:10.1109/INDIN.2006.275737 
[25] H. S. AbdelSalam and S. Olariu, "HexNet: HexagonBased Localization Technique for wireless Sensor Networks," IEEE International Conference on Pervasive Computing, Galveston, March 2009, pp. 1-6. doi:10.1109/PERCOM.2009.4912871
[26] J. Yi, J. Koo and H. Cha, "A Localization Technique for Mobile Sensor Networks Using Archived Anchor Information," Sensor, Mesh and Ad Hoc Communications and Networks, San Francisco, June 2008, pp. 64-72.

[27] http://www.decawave.com/ 